\title{
SE FAIRE / HACERSE + ATTRIBUT: UNE ÉTUDE CONTRASTIVE DE DEUX SEMI-COPULES PRONOMINALES
}

Introduction. Dans les corpus de traduction tels que les débats parlementaires de l'Union européenne (http://urd.let.rug.nl/tiedeman/OPUS/) on observe que se faire ne se traduit pratiquement jamais par hacerse et inversement, alors que le premier réflexe serait justement de considérer ces deux verbes comme des équivalents sémantiques. Certes, ce curieux constat peut avoir été biaisé par le corpus en question, ${ }^{1}$ mais il n'en reste pas moins que des exemples tels que (1 et 2) ne se traduisent pas par se faire et hacerse respectivement:

(1) La unidad política se hace efectiva ( $\rightarrow$ devient effective, réelle)

(2) La femme se fait belle belle $(\rightarrow$ se pone guapa)

Ce constat est en porte à faux avec les leçons de la littérature contrastive, qui nous apprend que hacerse correspond à se faire / devenir, même si d'autres équivalents de traduction sont encore mentionnés comme se rendre et, curieusement, paraître (voir Coste et Redondo 1965; Camprubi 1982:66-73; Bouzet 1990:252, §579). D’autre part, la littérature espagnole indique que hacerse s'insère dans un paradigme de verbes copules qui tous marquent le changement d'état, avec des nuances diverses (Porroche 1990; Bybee et Eddington 2006): volverse, ponerse, hacerse, llegar a ser, venir a ser ou acabar por ser. Il s'ensuit, logiquement, que hacerse, doit partager le terrain avec d'autres concurrents, ce qui laisse supposer que le spectre d'emploi de hacerse sera moins vaste que celui de devenir, qui fonctionne comme une sorte de verbe générique.

Dans cet article, il s'agira donc de faire ressortir les principales convergences et divergences dans l'emploi attributif de se faire et hacerse. En l'absence d'un corpus parallèle fiable et suffisamment grand, nous avons pris

1. Ne connaissant pas la langue source (qui peut d'ailleurs être une autre langue, l'anglais, par exemple), nous ne pouvions nous fier à ce corpus. 
principalement comme outil de travail deux corpus monolingues constitués de textes du $20^{\mathrm{e}}$ siècle, le Corpus del Español de Mark Davies et le corpus Frantext pour le français ${ }^{2}$ (complétés par des recherches sur Google, dont les résultats ont été soumis à des locuteurs natifs). Ces deux corpus sont plus ou moins comparables ${ }^{3}$ ce qui nous a permis de comparer des fréquences absolues d'une langue à l'autre. Dans un deuxième temps, nous avons fait appel à des locuteurs natifs pour vérifier quels emplois de hacerse (en contexte), de loin le plus fréquent, pouvaient effectivement être traduits par se faire, pour aboutir à une analyse contrastive plus robuste, notamment au niveau des convergences.

Au-delà de cet objectif purement descriptif, cette étude voudrait répondre à deux questions d'ordre théorique. Tout d'abord le statut du pronom et, par corrélation, sa nature analysable ou inanalysable. En d'autres termes, il est nécessaire de s'interroger sur la réflexivité ou l'intransitivité de la construction pronominale par rapport à la construction non pronominale:

(3) je me lave $[$ pronom réflexif $=\mathrm{COD}]<$ je la lave

(4) Il s'évanouit $<*$ Il l'évanouit

On peut observer que le pronom de apparaît comme un véritable pronom doté d'une fonction argumentale et référentielle, tandis que dans la forme réflexive apparaît comme une composante du lexème verbal. La construction pronominale tend donc à s'autonomiser par rapport à la construction transitive. A fortiori, c'est le cas avec hacerse et se faire, qui tendent à constituer des verbes copules ${ }^{4}$ inanalysables. En filigrane se pose la question de la lexicalisation et de la polysémie.

En outre, un autre intérêt théorique est suscité par l'apparition d'emplois évidentiels. Ainsi, le Diccionario de uso español mentionne comme equivalent de 'parecer' le verbe hacerse (hacérsele a alguien cierta cosa), tout comme Porroche (1990:116). Par exemple:

(5) Se me hace que no están muy bien avenidos

Côté français, Le Trésor de la Langue Française (TLF), signale que se faire + adj. signifie parfois 'commencer à être et paraître'. Il semble donc que hacerse et dans une moindre mesure se faire apparaissent dans les dictionnaires avec

2. http://davies-linguistics.byu.edu/personal/; http://www.frantext.fr/.

3. Le corpus Davies, à la différence de Frantext, comporte des écrits provenant d'Amérique du Sud qui diffèrent, dans certains cas, de l'usage qui en est fait dans la péninsule ibérique. Pour sa part, Frantext contient surtout des textes littéraires avec ce que cela implique: figure de style, métaphore, etc., donc forcément des interprétations diverses. Il faut ajouter que nous avons éliminé tous les exemples oraux de Davies, car Frantext n'en contient pas.

4. Ou encore semi-copules (Hengeveld 1992, Lamiroy et Melis 2005) ou pseudo-copules (Porroche 1990). 
deux emplois, l'un aspectuel ('devenir'), l'autre évidentiel. Ces observations éparses laissent transparaître un processus de subjectification.

Le plan de cet article sera comme suit. Nous fournirons d'abord un aperçu global des résultats de nos analyses de corpus (2), pour ensuite classer les différents emplois, combinant à la fois des critères sémantiques et morpho-syntaxiques, de manière hiérarchisée (3). Ces résultats seront ensuite mis en perspective à la lumière de l'autonomisation du tour pronominal et de la subjectification (4). ${ }^{5}$

1. Analyse quantitative: tendances globales. Nous présentons ci-après les résultats quantitatifs de nos études de corpus, impliquant des corpus monolingues. Ces premiers résultats donneront une vue très globale sur les rapports de hacerse et se faire, qui sera approfondie par la suite par le biais d'une étude plus qualitative.

Figure 1

$\begin{array}{ll}\text { Frantext }^{6} & \text { Davies } \\ \text { se faire } & \text { hacerse }\end{array}$

\section{$20^{\mathrm{e}}$ siècle}

Adj.: 7,45 (577) ${ }^{8}$

Adj.: 31,56 (487)

Sujets animés: $3,06(237)-41 \%$

Sujets animés:

Sujets inanimés: 4,72 (69) $-14,1 \%$

$4,39(340)-58 \%$

[dont 1,19 (92)

liés au domaine des animés]

$\mathrm{N}$

3,79 (293)

Sujet et attribut

animés: 1,71 (133)

$-45 \%$
Sujets inanimés:

27,4 (423) $-86,8 \%$

[dont 1,36 que (21), 3,43 infinitif (53) et (22)

liés au domaine des animés]

12,25 (189)

Animés: 4,34

(67) $-35,5 \%$

5. Cette étude s'inscrit donc dans l'analyse contrastive de faux amis (angl. cognates) dont on a souligné ailleurs tout l'intérêt pour les recherches sur la grammaticalisation (Lauwers, Vanderbauwhede et Verleyen 2010, sous presse).

6. Les corpus comptent respectivement 77406645 (Frantext) et 15426781 mots (Davies). En tout, le corpus de Davies compte 20.540.030 de mots dont 5.113.249 proviennent du corpus oral (chiffres fournis par M. Davies sous "corpus composition (texts)").

7. Pour les adjectifs attributs coordonnés, nous n'avons pris en considération que le premier adjectif. Nous avons cherché à éliminer ainsi la possible interférence de la coordination qui pourrait en effet relâcher les restrictions de sélection entre le verbe et l'attribut. Nous n'avons pas inclus les attestations de la locution se faire fort de.

8. Fréquence par million de mots. Les nombres absolus figurent entre parenthèses. 
Figure 1 (continued)

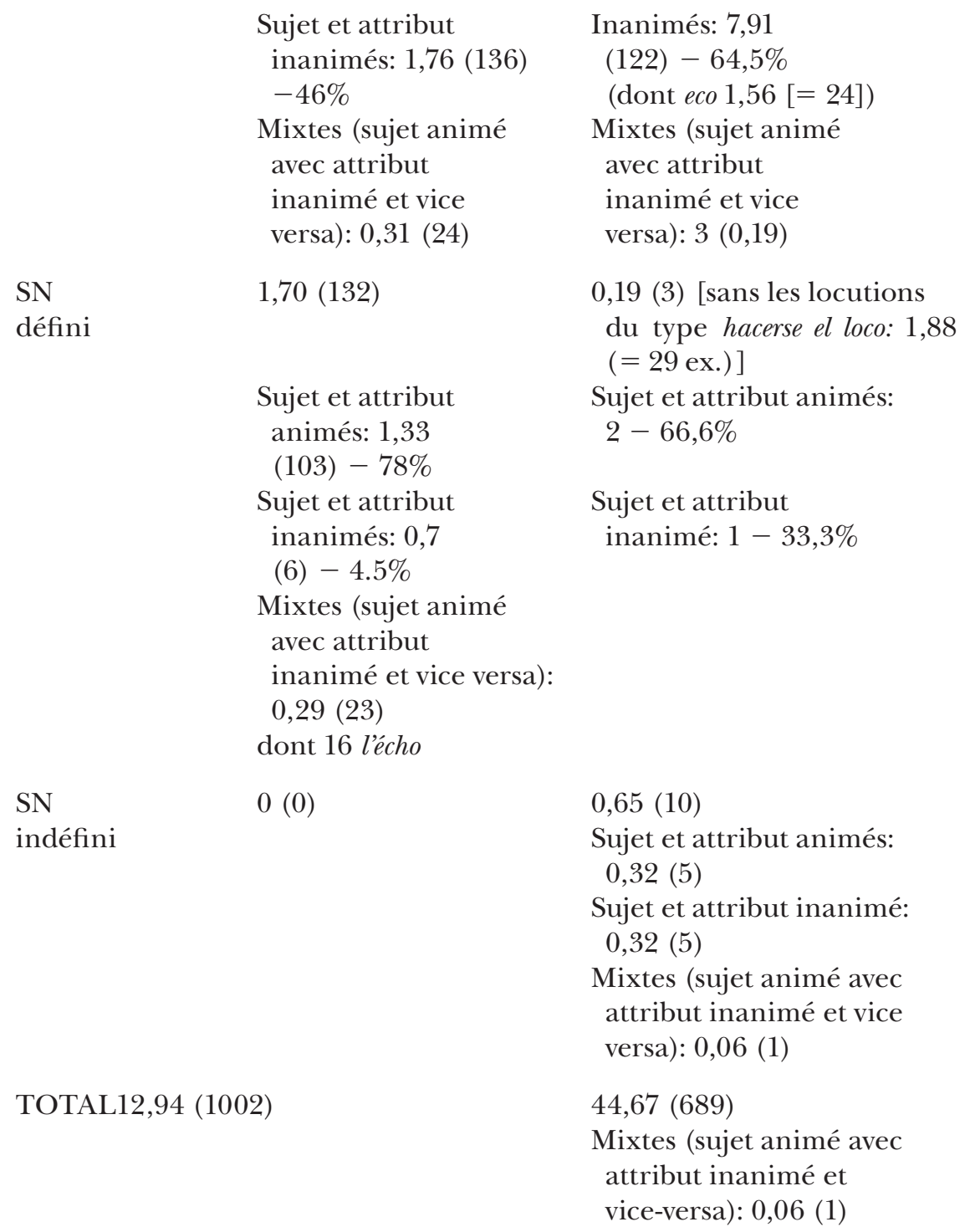

On constate que toutes les catégories majeures sont représentées en français et en espagnol, excepté la copule suivie d'un SN indéfini pour laquelle se faire ne fournit aucun exemple. Ensuite, la fréquence globale de hacerse + Adj. est presque quatre fois supérieure à la fréquence de se faire $(7,45$ vs $31,56)$. Il en va de même pour les noms nus, cela s'explique, mais seulement en partie, par une caractéristique plus générale de l'espagnol, qui accepte plus facilement des noms nus (= sans déterminant) en position 
d'attribut, indépendamment de la copule choisie (Van Peteghem 1993). L'on notera, que, curieusement, Davies n'est pas très bien représenté pour ce qui est des SN défini.

En ce qui concerne les types de sujets apparaissant aux côtés des deux verbes, on constate que hacerse + adj. ne se construit pas souvent avec des sujets animés (14,1 pour cent des cas), alors que se faire + adj. possède un sujet humain dans 41 pour cent des cas. Ces données et l'ensemble du tableau donnent à lire que l'idée de contrôle ou d'agentivité semble être plus souvent associée à se faire qu'à hacerse.

Replaçons ces observations dans le cadre de l'évolution diachronique:

Figure 2

Avant $1600^{9}$

Français

ADJ: $8,28(49)^{10}$

animé: $6,76(40)$

$81,6 \%$

inanimé: $1,52(9)$

$18,4 \%$
Espagnol

ADJ: 9,65 (350)

animé: 3,47 (126)

$36 \%$

inanimé: $6,18(224)$

$64 \%$

Si la fréquence de se faire + adjectif reste plus ou moins stable $(8,28$ occ. $>7,45$ occ.), hacerse + adjectif ${ }^{11}$ a gagné en importance $(9,65$ occ. $>$ 31,56 occ.). Ce qui frappe surtout, c'est que dans les deux langues, le recul est net pour les sujets animés (Fr. 81,6 pour cent $>41$ pour cent; Esp. 36 pour cent $>14,1$ pour cent).

2. Classification des emplois et étude détaillée. Pour la classification des emplois de se faire et hacerse, nous avons tenu compte de différents paramètres descriptifs. Pour garantir la comparabilité des emplois des deux verbes, il a été adopté un cadre sémantique dans lequel sont intégrés les aspects morpho-syntaxiques. Le premier niveau de la classification combine deux paramètres sémantiques:

(a) [ \pm changement d'état] ou [ \pm dynamique]: la phrase marque-t-elle un changement d'état (un devenir, cf. devenir) ou un simple état (cf. rester, être, sembler, etc.)?

(b) [ \pm contrôle]: le changement émane-t-il d'un sujet doté de volonté (un animé) qui a voulu le changement ou qui au moins a une certaine responsabilité dans ce changement? ${ }^{\text {12 }}$

9. Ici les deux corpus comptent respectivement 5915473 (Frantext) et 36.269.699 (Davies) mots.

10. N’ont pas été incorporés les locutions $i l$ se fait bon + inf. (3 ex.) et se faire fort de (7 ex.).

11. Nous écartons ici les $(\mathrm{S}) \mathrm{N}$, car le traitement de ce type de constituant est soumis à des divergences structurelles plus générales, qui, en plus ont fort évolué au fil du temps.

12. "[whether] the change comes about as a result of intentional effort, or whether the change occurs passively or unexpectedly" (Eddington 1999). 
La combinaison de ces deux paramètres donne lieu à trois catégories sémantiques: changement d'état contrôlé (3.1), changement d'état non contrôlé (3.2) et emploi adynamique (ou 'statique') (3.3). Il est à noter que l'opposition [ \pm contrôlé] est fonction de plusieurs sous-paramètres: la catégorie sémantique du sujet(animé vs inanimé, l'inanimé ne permettant pas le contrôle par définition) et la sémantique de l'adjectif, en combinaison avec le type de sujet (animé ou inanimé). Les trois catégories d'emplois (3.1, 3.2, 3.3) se déclinent en plusieurs sous-catégories où interviennent deux paramètres morpho-syntaxiques: la catégorie morpho-syntaxique de l'attribut(Adj/N/SN (in)défini), et la disponibilité de la construction transitive à attribut du COD. Ce dernier critère détermine la compositionnalité et l'analysabilité de la construction pronominale. Enfin, nous ferons aussi appel à quelques autres critères (morpho-)syntaxiques, tels que la possibilité d'insertion d'un complément datif, la disponibilité de la construction impersonnelle à extraposition et l'apparition de sujets de type phrastique, ainsi que des contraintes portant sur les temps.

Dans la présentation de chacun des emplois, priorité a été donnée aux données du français, qui constituent le cadre de la typologie; les analyses de hacerse y ont été chaque fois rattachées. Ce souci de parallélisme, inspiré par la volonté d'augmenter la lisibilité de l'analyse contrastive, nous a fait opter quelques fois un traitement en parallèle d'emplois qui n'étaient pas tout à fait identiques. Ces entorses à l'ordre de traitement attendu (qui ne concernent que le verbe hacerse) ont à chaque fois été signalées.

3.1. Le changement d'état contrôlé: ' $X$ fait qqch de sorte que $X$ devienne Y'. Le changement d'état contrôlé est associé à certaines caractéristiques, comme par exemple l'aspect animé du sujet, et peut être vérifié à l'aide de quelques tests distributionnels. ${ }^{13}$ Nous présenterons d'abord les emplois où la construction transitive correspondante est encore disponible (emplois réfléchis) (3.1.1), pour ensuite traiter des tours intransitivés (3.1.2).

3.1.1. Le changement d'état contrôlé réfléchi. L'appartenance à cette première catégorie d'emplois dépend de la vitalité de la construction transitive à attribut du COD correspondante, qui, en français, est désormais confinée à quelques emplois assez spécifiques. En espagnol, en revanche, l'emploi transitif attributif de hacer est encore largement attesté:

(6) Lo que lo hace entrañable es su práctica (Davies)

Cette construction se traduit en français non pas par faire, mais par rendre:

(7) Ce qui le rend attendrissant est sa pratique

Cependant, il reste des survivances de cet emploi transitif de faire, notamment:

13. Voir la liste classique présentée entre autres dans Willems (1981). 
(8) On la fait belle / C'est elle-même qu'elle fait belle

L'exemple (9) est susceptible de plusieurs transformations morphosyntaxiques qui incluent le pronominal réfléchi (a) et le passif périphrastique (b), ce dernier s'avérant toutefois douteux:

(9) (a) Elle se fait belle

(b) ??Elle a été faite belle

Un réseau de reformulations analogue existe pour les noms nus (il se fait moine), encore que parfois des glissements de sens s'observent d'une construction à l'autre:

(10) \# Ce n'est donc pas l'enseignement de l'art qui l'a fait peintre, mais un besoin vital d'exprimer dans le langage de l'art et de la peinture (Google) / une erreur du sort l'avait fait moine (Google)

(11) \# Il a été fait moine

Ainsi, on remarque que les phrases sont réinterprétées respectivement vers une influence déterminante (10) et un acte de nomination (11; cf. Il a été fait président).

Analysons maintenant plus en détail cette construction réfléchie, se faire + attribut, en nous arrêtant sur les différentes catégories attestées, c'est-à-dire l'adjectif (3.1.1.1) et le nom, tantôt sans déterminant (3.1.1.2), tantôt avec déterminant (3.1.1.3). Signalons d'ores et déjà que les noms précédés d'un déterminantseront traités en détail sous 3.1.2, étant donné que dans ce cas la construction transitive n'est plus disponible en français.

3.1.1.1. Adjectifs (Elle se fait belle). L'énoncé (9a) se caractérise par un changement superficiel et temporaire. En français (Frantext, 20 ${ }^{\mathrm{e}}$ siècle), les exemples se limitent à beau (25 exemples), joli (1) et désirable (1). Par contre, Google nous offre un choix plus large comme:

(12) Il se veut et se fait différent (alexis.blogs.com/francais/files/dossier_final_2.doc)

La volonté de la part du sujet dans y apparaît clairement puisque se veut fait écho à se fait. En espagnol, ce genre d'emploi semble réservé au verbe ponerse:

(13) me pongo guapa, la han puesto guapa

Par ailleurs la présence particulièrement importante de se faire beau (+ $^{+}$ joli/désirable) et son caractère plus ou moins isolé suggère que cet emploi est fortement lexicalisé. Avec le TLFi, on peut faire état d'une certaine spécialisation sémantique('se parer, s'apprêter, se maquiller'), ce qui est confirmé par le fait qu'on éprouve du mal à traduire cet emploi spécifique par hacerse en espagnol, qui, comme nous allons le voir, est pourtant un verbe qui a un spectre d'emploi plus large. 
3.1.1.2. Nom sans déterminant. Pour ce qui est des noms sans déterminant, deux cas de figure, assez spécifiques, doivent être distingués: le cas A, 's'auto-proclamer' et le cas B, 'devenir par un acte voulu'. Pour le cas (A), Frantext offre 9 exemples et Davies 2. Dans les deux cas, la reformulation transitive à attribut du COD est encore disponible, que ce soit avec des sujets inanimés ou animés ('nommer') et cela dans les deux langues:

(14) (a) Napoléon III, qui s'est fait président puis empereur (http://www .andrebarbault.com/justification_maitrises.htm)

(b) François Fillon demeure nettement supérieure à celle de l'homme qui l'a fait Premier ministre (www.liberation.fr/actualite/politiques/ 322315.FR.php)

(15) (a) El ex presidente ha puesto ilegalmente a su mujer en el puesto, él mismo se ha hecho presidente del partido peronista ('s'auto-proclamer') (www.cartelazo.blogspot.com/2008/06/amigos-de-la-libertad.html)

(b) Lo han hecho presidente

Cependant, selon le contexte, la construction espagnole peut se prêter à une deuxième interprétation, à savoir 'devenir président' (15) (c), où l'on peut tout au plus supposer une certaine volonté impliquée dans le changement, sans pour autant que le sujet ait un contrôle sur le résultat (qui dépend finalement d'autres personnes):

(16) (a) hay mucha gente que está esperando que usted se haga presidente de Real Madrid (ex. oral de Davies)

(b) */\#Beaucoup préfèrent que vous vous fassiez président $\rightarrow$ vous deveniez président

Ensuite, le cas B, assez proche de à vrai dire, s'en différencie cependant par le fait que le sujet a encore un certain contrôle sur le procès: se faire moine / hacerse monja. Certes, l'analyse s'avère délicate dans la mesure où, si elle est encore disponible, la construction transitive produit un effet archaïsant:

(17) C'est sa mère qui l'a fait poète et qui l'accompagne (immatériellement) jusqu'à ses lits d'amours ('il a été fortement influencé par sa mère') (www.kundera.net/biblio/VIE.php)

(18) subrayó que no abandonará el género romántico, porque es el que la ha hecho artista (www.elsiglodetorreon.com.mx/noticia/35673.crystalcritica-a-sergio-andrade.html)

Enoutre,commenousl'avonsdéjàremarqué,l'effetsémantiqueduverbetransitif, à savoir 'changement suscité par la pression / l'influence de quelqu'un / de quelque chose', ne se rattache plus que difficilement à la construction pronominale réfléchie qui signifie 'devenir par un acte voulu' et non pas 'exercer une pression sur soi-même pour devenir X'.

Dans les exemples du type B, le sujet (animé) de la phrase exerce un certain contrôle sur le changement d'état. Notons cependant que le corpus espagnol contient encore quelques exemples dans lesquels le nom nu attribut renvoie à un statut qui échappe complètement au contrôle du sujet 
(animé) de la phrase ou pour lesquels il est difficile de s'imaginer que le statut soit voulu: ${ }^{14}$

(19) Después bebió. Después se hizo borracho. Después perdió la dignidad, como dicen los carteles antialcohólicos (Davies) (*il se fit alcoolique)

(20) compró sus primeras acciones y anunció que si no se hacía millonario se tiraría del edificio más alto de Omaha (*sil ne se faisait pas millionnaire) (Davies)

La traduction par se faire + nom nu (qui nécessite le contrôle du sujet animé) est exclue, ce qui montre que, contrairement à se faire $+\mathrm{N}$ nu, hacerse + nom nu peut concerner des statuts qui échappent au contrôle du sujet. Mais ne resterait-t-il pas tout de même un relent de volition dans hacerse millionario/borracho? Quoi qu'il en soit, ce qu'il faut retenir ici, c'est que se faire $+\mathrm{N}$ est rétif à des changements dans lesquels toute volonté du sujet est absente, contrairement à hacerse $+\mathrm{N}$. Deux autres indices pointent dans cette direction. Ainsi, se faire ne convient pas dans les contextes où la volonté du sujet se voit annulée par l'intervention de facteurs externes. Comparez (a) y (b) de (21):

(21) (a) il est devenu médecin par vocation.

(b) ??il s'est fait médecin par vocation.

(c) il s'est fait médecin (car il l'a voulu)

(22) se hizo médico por una vocación que prendió en él desde la niñez (www .sld.cu/servicios/temas.php)

Dans le même sens, se faire ne convient pas à des statuts qui dépendent entièrement d'une nomination par des tiers, ce qui ne pose aucun problème pour hacerse (cf. l'exemple 16a, l'élection du président ne dépendant pas de lui-même). En somme, avec hacerse le contrôle exercé par le sujet animé peut être neutralisé, ou, du moins, affaibli à l'extrême, beaucoup plus que dans le cas de se faire $+\mathrm{N}$.

3.1.1.3. Hacerse + SN. Restent les SN 'pleins'. Comme seul le verbe hacerse prend des $\mathrm{SN}$ tout en restant analysable (= construction réfléchie), en toute logique, nous aurions dû traiter ici de hacerse. Or, pour la clarté de l'exposé, nous traiterons hacerse $+\mathrm{SN}$ en même temps que les emplois de se faire + SN (in)défini (cf. 3.1.2), qui eux, n'ont plus de correspondant transitif $^{15}$ (p.ex. Il s'est fait le champion des sans papiers).

14. Les exemples de hacerse se combinant avec un nom nu sans contrôle du sujet comportent bon nombre de cas où le français ne tolérerait jamais l'absence de l'article (indépendamment de la copule choisie). L'espagnol se montre en effet plus tolérant à l'égard des noms attributs nus (Van Peteghem 1993). Cela vaut notamment pour les pluriels nus, dont certains correspondent à des pluriels indéfinis en français (des jumeaux, des fiancés) et dans une moindre mesure frères, p. ex. Todos los hombres se hacen hermanos [Davies]).

15. A ce propos, on ne saurait confondre la construction non attestée *on l'a fait le porteparole des sans-papiers et la construction bitransitive à complément indirect $\{$ on / cela $\}$ a fait DE lui le porte-parole. . . 
3.1.2. Le changement contrôlé intransitivé (= non réfléchi). Les structures discutées ici ne se distinguent de celles présentées sous 3.1.1 que par l'absence d'une construction transitive correspondante:

(23) (a) Olivier se souvint de l'époque où il prenait le métro avec sa maman. Là, pour bénéficier de la gratuité, il devait se faire petit. (Frantext)

(b) $*\{$ Cela / Cette personne\} le fait petit.

Dans cet exemple, on peut paraphraser le sens de la construction comme suit: 'X subit un changement d'état dont X est lui-même responsable ou dans lequel il porte une part de responsabilité', mais se n'est plus conceptualisé comme l'objet (autonome) sur lequel porte la transformation, c'est-à-dire le patient. En outre, ce changement, voulu, n'est pas pour autant permanent et n'affecte pas les caractéristiques intrinsèques du référent. ${ }^{16}$ Cet emploi est l'emploi de base de se faire + adj. (3.1.2.1) et de se faire + SN défini (3.1.2.2) avec des sujets animés. Nous terminerons par quelques remarques sur les $\mathrm{SN}$ indéfinis (3.1.2.3). A chaque fois, nous y rattacherons les emplois correspondants de hacerse. Avouons que ce traitement-inspiré par un souci de parallélisme dans l'exposition-ne reflète pas vraiment l'analyse syntaxique, dans la mesure où hacerse + Adjectif / SN reste analysable, puisque la construction transitive est toujours disponible.

3.1.2.1. Se faire + Adjectif. Dans les emplois de se faire + adjectif, deux cas de figure peuvent être distingués, l'un étant plus fréquent (B; 103 ex.; $10,8$ pour cent $)^{17}$ que l'autre (A; 55 ex.; 5,8 pour cent):

(A) Adjectifs qui renvoient aux apparences physiques (au sens large) d'une personne, par exemple, l'énoncé .

(B) Adjectifs qui réfèrent à un état d'âme temporaire ou à un air pris par le sujet:

(24) (a) elle se fit méchante (Frantext, Chabrol)

(b) \# elle devient méchante

Pour le cas (A), tout d'abord, l'énoncé (a) montre que le sujet contrôle le changement et que ce changement, temporaire, est moins intrinsèque qu'avec devenir. ${ }^{18}$ Les adjectifs relevés dans le corpus Frantext sont petit

16. On peut signaler d'autres propriétés, par exemple la présence de toutes les autres personnes, je me fais, tu te fais, il se fait, etc.

17. Dans l'analyse détaillée des emplois avec un attribut adjectival, nous avons fait abstraction de quelques exemples relevant du jargon philosophique. Ceux-ci ont bel et bien été incorporés dans les calculs statistiques.

18. Dans certains cas, la commutation avec devenir n'entraîne pas nécessairement un glissement vers un changement intrinsèque, les deux interprétations étant disponibles: La grenouille se fait grosse = 'La grenouille devient grosse' ('a pris du poids' / 'se fait grosse'). 
(22), rare (22), minuscule (2), nu (2), léger, discret, furtif ('discret') et fuyant. ${ }^{19}$ En bref, le type (A) est représenté par un ensemble limité de lexèmes parfois très fréquents. Le type (B), que l'on voit représenté dans l'exemple (24) (a), frappe par le grand nombre de lexèmes différents (type frequency très élevée) - pas moins de 68 dans Frantext—qui s'y prêtent comme humble (7), attentif (3), aimable (4), etc.

En plus des sujets animés à proprement parler qui relèvent du type B, on trouve 92 exemples de noms inanimés qui entretiennent un rapport avec le domaine de l'animé, que ce soit par voie métonymique (<voix >, $<$ regard $>$, <visage $>$, <gestes $>$, <esprit, âme $>$ ) ou par le biais d'une personnification, ou encore, parce qu'il s'agit de noms collectifs à référence humaine (p.ex. village):

(25) Sa voix se fit grave

En d'autres termes, le sujet n'est pas proprement animé, mais subit un changement contrôlé 'indirectement' par un être humain. La fréquence assez élevée de cet emploi montre que se faire se prête à merveille à ce "contrôle indirect", notamment pour introduire un passage en discours direct (Sa voix se fit grave: “. ..").

Il convient d'attirer encore l'attention sur un effet de sens, voire une inférence pragmatique, qui apparaît parfois dans les exemples du type (B). En effet, étant donné que le changement d'état (d'âme / de caractère) voulu est caractérisé par la momentanéité, on se rapproche facilement de l'apparence ou de l'attitude feinte:

(26) Les femmes qui venaient chercher leur enfant se transformaient en mères à vue d'œil. Elles prenaient un air responsable, leurs gestes se faisaient maternels ou feignaient de l'être. Une bourgeoise aussi digne que ridicule me fit sourire. (Frantext, Jardin. A, 1986) (Attitude feinte)

Notons que devenir ne donne pas lieu à ce genre d'inférences.

3.1.2.2. Hacerse + Adjectif. En ce qui concerne l'emploi (B) que nous venons de distinguer, il est improbable de trouver en espagnol hacerse + adj. avec le sens d'un changement temporaire contrôlé par le sujet:

(27) (a) Pierre se fait désagréable. 'prend un air désagréable'

(b) \#Pedro se hace desagradable. 'son caractère est devenu désagréable' (intrinsèquement)

En effet, quand on cherche à traduire les exemples de se faire du type (B), on constate que hacerse ne se combine pas avec des adjectifs désignant des états d'âme ponctuels, par exemple *Se hizo suplicante ('Il se fit suppliant').

19. En plus, 6 exemples philosophiques concernent des emplois de se faire où Dieu et l'homme sont considérés comme des entités capables de se créer elles-mêmes. 
L'espagnol demande plutôt ponerse (changement temporaire) ou volverse (changement temporaire ou intrinsèque): se volvió irónico, desagradable, amable, etc. Il n'empêche qu'on peut trouver de rares exceptions où hacerse tend vers une interprétation temporaire:

(28) No hay que dar por conocido lo que no lo es. (Y) Tal vez por eso el filósofo socrático se hace desagradable para todo el mundo (CRAE) ('se montre', 'apparaît aux yeux de', 'prend un air')

Mais, en principe, hacerse exprime plutôt des changements intrinsèques, qui se traduisent par devenir ou se rendre (le plus grand roi d'Israël ne crut pas se rendre désagréable à Dieu, en lui adressant des prières; Google) et non plus par se faire. Quant aux sujets qui renvoient par voie métonymique à un sujet humain, comme la voix, le ton, les gestes, etc., ceux-ci sont susceptibles d'être traduits par hacerse et de garder le sens d'un changement temporaire: ${ }^{20}$

(29) (a) La voix de Nicole se fit claire

(b) La voz del locutor se hace clara (Davies, ex. provenant du Paraguay)

Sur ce point, ces exemples de hacerse s'alignent donc sur les emplois avec un sujet inanimé, où hacerse connaît un emploi très large et où il s'utilise sans problème pour marquer un changement éphémère:

(30) $\{$ El viento / la luz $\}$ se hizo desagradable

Quant au type (A), c'est-à-dire les changements physiques voulus et temporaires ou superficiels (non intrinsèques), se faire peut se traduire par hacerse (bien qu'un verbe intransitif y soit souvent préférable):

(31) (a) se hace pequeño, la rana se hace (más) grande/gruesa que el buey

(b) il se fait petit, la grenouille se fait plus grande que le bœuf

De la comparaison se faire / hacerse + adjectif il ressort donc que la distribution des deux verbes dans le domaine de [+animé] et [+contrôle] est très inégale. On retiendra pour se faire notamment l'absence du changement voulu de type intrinsèque, ce qui n'était pas le cas dans les stades plus anciens de la langue (Frantext, exemples d'avant 1600):

20. Si on cherche à traduire les exemples du corpus espagnol, on constate qu'un des exemples est néanmoins traduisible par se faire, bien qu'il exprime un changement d'état voulu et intrinsèque: $X$ se hace responsable /X se fait responsable. L'hypothèse qui s'impose est que les adjectifs de ce type (despótico / sedentario / democrático), qui désignent des propriétés intrinsèques et voulues, se construisent avec se faire parce qu'ils s'emploient à la fois comme nom et comme adjectif. Signalons à ce propos que les noms nus, accompagnés d'une expansion-qui en souligne le caractère nominal-apparaissent sans problème aux côtés de se faire (sédentaire, despote, responsable (syndical)). Notre hypothèse se voit corroborée par le fait que, par exemple, se faire despotique (aucun exemple sur Google) est beaucoup moins acceptable que se faire despote, qui se confond avec la forme nominale (quelques dizaines d'exemples sur Google). 
(32) (a) Il n'y a chose plus profitable à l'homme pour se faire riche et contregarder son bien que se faire aymer d'un chacun ['fabriquer sa fortune grâce à son travail par exemple']

(b) Les Français polis se font meilleurs

Ces exemples ne sont plus acceptables de nos jours. ${ }^{21}$ Hacerse, de son côté, se signale par la quasi-absence de l'interprétation 'prendre un air, se montrer'.

3.1.2.3. Hacerse / se faire + SN définis. Passons aux SN définis. Rappelons que, curieusement, le SN défini est beaucoup plus fréquent en français qu'en espagnol (cf. tableau fig.2):

En effet, on ne compte que trois exemples non locutionnels dans le corpus de Davies (dont l'un se construit avec un attribut inanimé), comme (33):

(33) Sport, el Juventus se apagó muy pronto y dejó que el Madrid se hiciese el dueño del juego. (Davies)

(34) Enfant, il avait déjà besoin de recréer physiquement les personnages de ses lectures préférées au point que sa mère se faisait sa complice . . . (www.gerard-sety.com/carriere.html)

Les 29 autres exemples relèvent tous de la locution hacerse el $\mathrm{N}$ (p.ex. hacerse el tonto), l'équivalent de la locution attributive faire le $\mathrm{N}$ 'se comporter comme', comme dans faire le fou (Lauwers 2008). Les exemples locutionnels de hacerse $+\mathrm{SN}$ se distinguent par le fait que la construction transitive n'est plus disponible et qu'ils n'admettent aucun déterminant autre que le déterminant défini. Comparez: *se hace su tonto, *se hace este tonto. La fréquence plus élevée de se faire $+\mathrm{SN}$ défini ${ }^{22}$ tient à deux facteurs. Le premier facteur est la concurrence de la locution hacerse el $N$, ce qui fait que tout SN défini apparaissant aux côtés de hacerse risque d'être interprété comme constituant une locution. Le deuxième facteur se fonde sur le spectre d'emploi plus large du nom attribut sans déterminant en espagnol (cf. Van Peteghem 1993). On remarque en effet que sur 132 exemples français, 16 se traduisent par un $\mathrm{N}$ sans article, dont 5 fois eco $(<$ l'écho). L'espagnol apparaît comme plus tolérant à l'égard des noms attributs sans article ( $h a$ cerse eco). Cela n'a rien à avoir avec la valence spécifique des verbes se faire/ hacerse, mais avec le statut du nom nu en espagnol. En revanche, si le référent est vraiment unique-ce qui va souvent de pair avec des modificateurs supplémentaires, comme dans hacerse cómplice de / hacerse el complice activo de ...- - ou si l'on veut insister sur le caractère défini du SN, nos informateurs traduisent par un SN défini:

21. Certes, le corpus Frantext (corpus littéraire) contient encore quelques très rares exemples de changements intrinsèques voulus, mais ceux-ci sont rejetés carrément par nos locuteurs natifs, à l'exception de trois exemples-certes, archaïsants-dont afin qu'ils [= les riches] se fissent pauvres, où le doute est permis quant au statut adjectival ou nominal de l'attribut. Cette observation rejoint la note précédente.

22. Les quelques rares exemples espagnols construits avec un sujet animé se traduisent sans problème par se faire; pour l'unique exemple inanimé, par contre, devenir s'impose. 
(35) (a) Il voulait appeler les dirigeants soviétiques à se faire les héros d'une résistance nationale contre l'envahisseur (Frantext)

(b) quería llamar a los dirigentes soviéticos a que se hicieran los héroes

En plus des deux facteurs évoqués ci-dessus, il convient de signaler un troisième facteur qui pourrait expliquer le contraste en termes de fréquence entre hacerse et se faire $+\mathrm{SN}$ défini. A y regarder de plus près, on note dans le corpus français une certaine tendance à la lexicalisation, ou, du moins, à la constitution d'un paradigme d'expressions plus ou moins synonymiques autour de l'idée "défenseur de X"23 (p.ex. se faire le champion, l'avocat, le défenseur, le porte-parole de). Le plus souvent, la caractérisation est moins intrinsèque dans la mesure où il s'agit de quelqu'un qui s'approprie le statut de défenseur. C'est d'ailleurs pourquoi la traduction par la construction hacer de ('se comporter comme') apparaît parfois quand on cherche à traduire ces exemples. Le sujet est animé et contrôle le procès. Plus de la moitié des exemples du corpus Frantext peuvent être rattachés à ce type.

3.1.2.4. Hacerse / se faire $+\mathrm{SN}$ indéfinis. Restent les $\mathrm{SN}$ indéfinis, dont on ne trouve aucune attestation avec se faire dans Frantext, mais bien dans Google:

(36) la décision d'implantation de la mosquée a été prise par le nouveau maire, qui se faisait un champion de la démocratie dans la ville (www .books.google.be/books?isbn=2738422276)

Les SN indéfinis sont un peu plus fréquents avec hacerse, au point que leur fréquence dépasse celle des SN définis (abstraction faite des locutions du type hacerse el tonto): 0,65 vs 0,19 occ. par million de mots. En outre, ils se répartissent de manière égale dans le domaine de l'animé et de l'inanimé comme le montre la et les exemples suivants:

(37) (a) en pocos años se hizo un viejo

(b) El trabajo, la familia, los amigos, todo se hacía una rutina insoportable

Nous n'avons trouvé que deux exemples où toute forme de volonté semble absente:

(38) (a) Babilonia se hizo una ciudad-estado independiente.

(b) para que Daniel, el Mochuelo, se hiciera un hombre en la ciudad, para que progresase y no fuera como él.

Rappelons encore, pour terminer, que hacer admet encore des SN (in) définis comme attributs de l'objet, ce qui rend la construction pronomi-

23. Le fait que les noms qui apparaissent comme SN attribut prennent parfois un sens assez spécifique, montre que cette construction, qui demande toujours un complément en $d e$, tend à devenir une construction sui generis. Il serait intéressant d'examiner si elle ne s'est pas formée d'après l'expression se faire l'écho de. 
nale parfois analysable. En toute logique, nous aurions donc dû traiter ces exemples analysables (à sujet animé et volitifs) sous 3.1.1., parmi les emplois réfléchis.

3.2. Le changement d'état non contrôlé, passif: ' $X$ devient $Y, X$ subit un changement qui le fait passer de $\mathbf{X}$ à $\mathbf{Y}$ '. Les emplois classés sous 3.1 se caractérisaient par la présence d'un sujet animé contrôleur, doté de volonté. Cet emploi, qui est encore largement attesté pour se faire (à témoin la forte présence de sujets animés dans les emplois adjectivaux: 41 pour cent), est minoritaire pour hacerse ( $<14,1$ pour cent pour les adjectifs). En outre, hacerse se prête facilement à des lectures non agentives, en dépit de la présence d'un sujet animé (tant pour les attributs adjectivaux que nominaux), comme nous l'avons vu. Tel emploi n'est pas disponible pour se faire, à l'exception des exemples lexicalisés listés sous 3.2.2. de type Pierre se fait vieux.

Il est temps d'aborder les cas où le sujet n'est plus agent et où se faire entre donc en concurrence avec devenir. C'est ici que l'analyse de se faire devient très délicate. Si l'analyse distributionnelle fera ressortir des tendances assez nettes, il n'en reste pas moins que parfois se faire ne semble être qu'un synonyme de devenir que, certes, on situe dans un registre de langue plus élevé.

Plusieurs caractéristiques président à cet emploi:

—il y a un changement d'état, donc "X subit un changement qui le fait passer de X à Y"

—le sujet n'a pas de contrôle sur le procès, que le sujet soit inanimé ou animé (dans ce dernier cas, le contrôle est neutralisé par l'adjectif)

-le pendant transitif "simple" de la construction pronominale n'existe pas. En réalité, le pronom réflexif fonctionne comme un marqueur syntaxique de clôture du procès (Melis 1990:63-64).

Dans un premier temps, nous allons analyser la distribution de se faire (et de hacerse) avec des sujets inanimés, en nous basant sur le sémantisme des adjectifs qui apparaissent en fonction d'attribut (3.2.1). Ensuite, il sera question des exemples impliquant des sujets animés (3.2.2), qui, pour se faire, témoignent d'un processus de lexicalisation. Nous terminerons par quelques remarques sur les attributs nominaux (N et SN) (3.2.3).

3.2.1. Avec un sujet inanimé: l'eau se fait rare. Il ressort de la figure 1 que la construction hacerse + adjectif avec des sujets inanimés est six fois plus fréquente que dans le cas de se faire. Ce constat est confirmé par le fait que pour un grand nombre d'adjectifs et de champs sémantiques la traduction ${ }^{24}$ de hacerse par se faire est tout simplement impossible. Pour

24. La traduction d'exemples littéraires en français s'est révélée parfois très ardue. Hors contexte, la traduction par se faire paraissait parfois bizarre, impression qui disparaissait aussitôt que nous nous trouvions confrontés à des exemples contextualisés provenant de Google. En outre, comme se faire relève d'un registre plus soutenu que devenir (et hacerse) et que, de ce fait, il est moins fréquent—et de moins en moins, en fait—, on 
faire ressortir les différences entre les deux verbes et pour conférer malgré tout une certaine robustesse à cette analyse délicate, nous avons regroupé les convergences (3.2.1.1) et divergences (3.2.1.2) en champs sémantiques, commençant par les domaines où se faire et hacerse se chevauchent. Avouons d'emblée que l'analyse proposée n'épuise pas toute la question dans la mesure où-pour les raisons évoquées ci-dessus-elle ne permet pas de circonscrire avec netteté le champ d'application du verbe se faire. Il n'empêche qu'il en ressort nettement une perspectivation subjective (3.2.1.3), notamment dans le cas de se faire.

3.2.1.1. Convergences: se faire / hacerse + adj. De l'analyse des adjectifs émergent 6 champs sémantiques où se faire et hacerse convergent. Ces 6 catégories représentent 67,8 pour cent des exemples relevés dans Frantext. La pertinence de ces catégories a été vérifiée à l'aide de traductions: les exemples de hacerse où la traduction par se faire était possible rentrent tous dans les 6 catégories. Comme nous avons préféré ne pas nous lancer dans l'analyse d'effets littéraires ${ }^{25}$ ces pourcentages auraient pu être plus élevés encore. La pertinence de nos catégories ressort aussi de l'analyse des exemples trouvés sur Google. Ainsi, une recherche de "se fait très" donne uniquement des exemples des trois premières catégories. Regardons de plus près les 6 catégories. Nous mentionnons le nombre d'exemples entre parenthèses:

Type A. Adjectifs indiquant "l'accessibilité (accrue / moindre) à la perception”: Fr. rare (43), sensible (3), visible (3), précis (3), opaque (3), évident (3), nombreux (2), discret (2), clair (2), etc.; Esp. público (22), evidente, obvio, visible, mudo, ensordecedor, palpable, tangible, etc. En espagnol, la perception s'étend aussi à l'aspect cognitif ou épistémique (indiscutible, indudable. . . ). Avec les adjectifs de couleur, la traduction de hacerse par se faire est possible chaque fois que l'adjectif renvoie à l'accessibilité du référent à travers la perception visuelle (degrés de luminosité): Fr. (ciel) noir, (lumière) pâle, sombre (3), obscur, crépusculaire, gris; Esp. rosa, negro. En tout, les adjectifs du type A fournissent 37,1 pour cent des 248 exemples de se faire impliquant des sujets inanimés. Pour l'espagnol, 31 pour cent (131 exemples sur un total de 423 exemples) sont du type A.

Type B. Adjectifs signifiant 'difficile' ou 'insupportable' (vs 'facile'), éventuellement dans un mouvement qui va crescendo, une spirale négative: Fr. difficile, intenable, intolérable, blessant, oppressant, lourd (6), etc.; Esp. dificil (5) / fácil (1), insoportable (10), insostenible (2), complejo (2), penoso (1), incierto (el paso) (1), crítico (1), doloroso (1), etc. Les exemples de ce type sont très nombreux: Frantext 17 pour cent; Davies 13 pour cent. Contrairement

note des différences d'acceptabilité d'un locuteur à l'autre. D'autre part, nous avons été confrontés à certains adjectifs comme évident, dur, abondant et suffisant qui arborent des effets de polysémie ayant des répercussions sur l'emploi de se faire.

25. Pensons aux personnifications (les eaux se font accueillantes) et aux métaphores (la nuit se fait duveteuse) (exemples de Frantext). 
à ce que laissent penser ces pourcentages, hacerse est beaucoup moins restrictif sur ce point que son homologue français dans la mesure où hacerse se combine aussi avec les antonymes (mais beaucoup plus rarement): $L a$ vie se fait difficile / ?? facile vs La vida se hace dificil / fácil.

Type C. Adjectifs aspectuo-temporels exprimant la durée (et ayant un impact négatif sur l'expérienceur): Fr. + Esp.: largo/long, interminable, eterno/éternel, angosto; corto/court.

Curieusement, une fois de plus se faire est seulement possible si l'adjectif aspectuel indique une propriété qui a un impact négatif sur l'expérienceur (qui, par ailleurs, peut être exprimé par un datif en espagnol, mais pas en français: la película, se me ha hecho larga.). Comparez:

(39) *L'attente se fait courte; le médecin m'appelle après 10 minutes

En revanche, si court apparaît dans un contexte où c'est plutôt la courte durée qui a un impact négatif, court peut se combiner avec se faire:

(40) La nuit se fit courte. C'est à grand-peine que nous nous levâmes. (ex. construit)

Dans tous les cas, ces adjectifs renvoient à une expérience subjective du temps dans la mesure où les unités temporelles ne changent pas intrinsèquement. Ce qui change, c'est la perception par l'expérienceur.

Type D. Crescendo / haut degréde saillance

-le référent du sujet devient 'saillant' (dans un sens ou un autre): Fr. aigu (attention), prépondérant (rôle de conseil), telle que + intensif (peur), central (facette); Esp. importante, dominante, preponderante, prominente, predominante. .

-le référent du sujet atteint le degré maximal, éventuellement à travers un processus qui va crescendo: Esp. definitivo, absoluto; Fr. emplois archaïsants: définitive (séparation), totale (aliénation), éterne),??complet (nuit), ?? intact (malheur)

Type E. Adjectifs déontiques: Fr. impérieux, urgent, impératif; Esp. preciso ('indispensable'), necesario, innecesario, indispensable, imprescindible, inevitable, perentorio, urgente, pertinente, vital.

Les adjectifs déontiques sont particulièrement fréquents avec hacerse (25,9 pour cent). Dans Frantext, leur nombre est limité à 5 (2 pour cent). Il n'empêche que la traduction des 81 exemples espagnols par se faire est toujours possible. Il s'agit toujours d'une nécessité intrinsèque ${ }^{26}$ qui n'émane pas d'une instance institutionnelle (externe), comme avec obligatorio, le seul exemple où la traduction par se faireest bloquée:

26. L'on remarquera que se faire ajoute une nuance que l'on ne trouve pas avec devenir. Par exemple, dans la guerre se fait inévitable, la guerre a un rôle plus 'actif' dans la prédication que dans la guerre devient inévitable. C'est que dans se faire on trouve encore un relent d'activité hérité de son sémantisme d'origine. Il s'ensuit que se faire met en relief, plus que devenir, le caractère urgent, inévitable de la chose, qui en quelque sorte s'impose. 
(41) la pena de muerte se hace obligatoria vs *La peine de mort se fait obligatoire

Comparez à:

(42) la guerre se fait inévitable

Type F. Adjectifs marquant la surprise éprouvée par l'expérienceur (l'observateur se trouve interpellé, car le référent produit un effet bizarre, se présente comme bizarre):Fr.: bizarre, mystérieux, étrange, singulier, etc.; Esp.: singular, normal, misterioso. Assez souvent, l'étonnement est lié à la perception auditive ou visuelle. Ici encore, les antonymes ne passent pas la rampe avec se faire, contrairement à hacerse: *quelque chose se fait normal.

Les 6 catégories qui précèdent couvrent presque 68 pour cent des exemples de Frantext et tous les exemples du corpus espagnol traduisibles par se faire, à 10 exemples près, qui n'appartiennent pas à une des catégories mais qui restent malgré tout traduisibles: accessible, obsolète, impénétrable, frénétique, multilatéral, conservateur, normative, politique, varié, ${ }^{27}$ tranquille. En voici un exemple:

(43) les nuits se font tranquilles (= tranquilas)

3.2.1.2. Divergences. Du côté des divergences, on a pu relever cidessus que la traduction des antonymes des catégories $\mathrm{B}$, C et $\mathrm{F}$ (fácil, normal, etc.) s'avère impossible. En outre, on a vu que la traduction de l'espagnol en français se révèle difficile, voire impossible pour les champs sémantiques de la perception cognitive (épistémique) et de la couleur (quand celle-ci n'est pas liée à l'accessibilité à la perception).

En plus, l'étude de nos deux corpus monolingues, combinée à l'examen des possibilités de traduction, ont permis d'identifier aussi des champs sémantiques couverts par hacerse où se faire ne met jamais le pied. Il s'agit d'adjectifs qui désignent des propriétés objectives telles que la forme ou la taille (redondo, grande, pequeño, corto, vertical, extensible, profundo, extensif), la consistance ou la matière(duro (rodilla), sólido, liquido, soluble, inestable, selvático ('boisé/poilu'), salobre ('saumâtre'), fuerte, firme, vulnerable, et des propriétés esthétiques appliquées à des inanimés comme hermoso et ostentoso. Il n'empêche que quelques adjectifs que l'on rattacherait spontanément à ces catégories admettent se faire sans que l'on puisse en donner une explication satisfaisante: souple (cf. aussi flexible) et fluide (circulation, lecture, conversation, etc.). Quant à insuffisant, tout ce qu'on peut dire, c'est que dans certains exemples on note une plus forte implication de l'observateur (qui ressent un impact négatif):

(44) \{Son salaire / l'espagnol\} se fait insuffisant pour se tirer d'affaire aux USA ('il a pu s'en rendre compte')

27. La liste de participants se fait variée ('se présente comme', adynamique). 
D'autres adjectifs ne semblent pas pouvoir se combiner avec se faire, sans qu'on puisse vraiment les rattacher à une classe sémantique particulière, qu'il s'agisse d'adjectifs gradables (divertido/amusante) ou non gradables (masculino/masculin).

Signalons, enfin, une différence qui a trait à la catégorisation grammaticale. Hacerse se combine facilement avec des adjectifs dérivés de participes comme abierto, divertido, ou encore, avec des adjectifs déverbaux en -able (ou -ible) peu lexicalisés (Leeman 1992). Quant à ce dernier aspect, comparez * se faire / hacerse penetrable à se faire / hacerse impenetrable. Là encore se faire se montre plus restrictif que hacerse.

3.2.1.3. Bilan: une perspectivation subjective. Si l'on met ces observations distributionnelles en perspective, il faut en conclure que la combinaison d'un sujet inanimé et de se faire suivi d'un adjectif suppose une forte implication d'un expérienceur (ou conceptualisateur; Langacker 1990) humain, qui peut coïncider avec le sujet parlant, mais pas nécessairement (par exemple, si la phrase se trouve à un temps du passé). On peut dès lors parler de subjectification, que l'on peut définir, d'après la définition Traugott-pour d'autres approches, voir Stein et Wright (2005) et Athanasiadou et alii (2006) — comme un processus au cours duquel "meanings tend to become increasingly situated in the speaker's subjective belief state or attitude toward the proposition" (Traugott 1989:31). En l'occurrence, cette perspectivation subjective se manifeste de la manière suivante:

(a) se faire + adjectif marque une implication subjective sur le plan de la perception (notamment visuelle), plus particulièrement un changement portant sur l'accessibilité sensorielle: quelque chose devient plus ou moins visible, audible, etc. pour l'observateur, mais le changement n'affecte pas le référent même. Dans le cas de hacerse, la perception visuelle s'étend à la perception cognitive.

(b) se faire a une nette préférence pour les adjectifs-aspectuotemporels et autres-qui marquent une propriété perçue comme négative ou désagréable par un expérienceur, au point qu'elle devient insupportable

(c) l'implication subjective est induite par la présence de prédicats déontiques, qui signalent une nécessité qui s'impose d'elle-même à un expérienceur.

C'est donc la subjectivité qui présiderait à la distribution de se faire (et dans une moindre mesure de hacerse). Le changement ne porte plus sur le référent, mais concerne le rapport entre le référent et l'expérienceur (ou conceptualisateur). Notamment dans les (exemples espagnols des) types B et $\mathrm{C}$, on ne sent plus ou à peine le changement d'état, sauf s'il y a des éléments contextuels qui mettent l'accent sur un déroulement temporel qui rend un changement d'état (d'âme) possible. On comprend maintenant mieux pourquoi se faire a une distribution si capricieuse (indépendamment des problèmes liés au type de corpus et au caractère parfois archaïsant du 
verbe). Ce qui distingue finalement se faire de devenir dans le domaine du changement d'état non contrôlé, c'est l'implication d'un expérienceur ou conceptualisateur qui reste implicite et qui correspond le plus souvent au sujet parlant.

En revanche, le spectre d'emploi de hacerse est beaucoup plus large, puisqu'il ajoute à un noyau constitué d'adjectifs liés à la perspectivation subjective où se faire et hacerse se côtoient, des extensions, y compris vers des adjectifs objectifs (forme, matière, etc.) et des adjectifs moins prototypiques en termes d'adjectivité (participes, adjectifs non gradables, adjectifs déverbaux en -able/-ible peu lexicalisés).

3.2.2. Sujets animés (sans contrôle): Pierre se fait vieux. Si la quasitotalité des exemples du changement sans contrôle contiennent des sujets inanimés, force est de constater que tant se faire que hacerse admettent aussi des sujets animés (humains notamment), mais pas au même degré.

Regardons d'abord les données du français. Les adjectifs suivants sont attestés: vieux (29), grand (1), chauve (1), gras/poussif, aveugle et médiocre. Apparemment, cette combinaison est loin d'être évidente pour se faire, comme le montre le fait que plusieurs exemples de Frantext sont rejetés par les locuteurs natifsque nous avons consultés(*se faire chauve). Il s'agit en effet d'un emploi ancien, comme le montre aussi l'inacceptabilité de l'exemple suivant, datant de la fin du $17^{\mathrm{e}}$ siècle:

(45) *Un enfant qui se fait grand, il s'est fait grand en très peu de temps (Dictionnaire de l'Académie française, 1692)

En définitive, seul vieux persiste et signe, au point qu'on pourrait y voir un autre emploi lexicalisé, après se faire beau/joli (cf. 3.1.1.1). On aurait donc deux cas de lexicalisation (avec des sujets animés), l'un dans le domaine du contrôlé, l'autre dans le domaine du non contrôlé. L'identification de ces vestiges d'un état de langue ancien renforce l'hypothèse selon laquelle se faire + sujet animé est une combinaison qui n'a cessé de régresser depuis le $17^{\mathrm{e}}$ siècle (cf. fig. 2).

En revanche, en espagnol, la combinaison d'un sujet animé avec un prédicat qui n’admet pas le contrôle est tout à fait courante:

(46) Ningún insecto puede ser peor que el hombre que se hace vil / *l'homme se fait vil (prolibertadprensa.blogspot.com/. . ./los-sopranojuan-gonzlez-febles.html)

Comme hacerse autorise aussi les changements intrinsèques avec contrôle du sujet (cf. 3.1.2), il s'ensuit que dans certains cas, hacerse peut être ambigu entre une lecture avec ou sans contrôle:

(47) (a) Mi abuela se hace pequeña porque ha visto a un ogro y tiene mucho miedo. (= procès controlé)

(b) Mi abuela se hace pequeña con la vejez. (= processus naturel, qu'on subit, cf. se hace vieja). 
Il ressort de cela que l'analyse classique de hacerse est erronée, ou du moins, incomplète. Ainsi, on associe toujours le verbe hacerse à l'expression du changement voulu (cf. Camprubi 1982; cf. introduction), description qui, en fin de compte, s'appliquerait mieux à se faire . . . Et ce d'autant plus que la fréquence globale des sujets animés reste inférieure à 15 pour cent dans le corpus hacerse (cf. fig. 1). En plus de l'angle d'attaque choisi, qui cherche à différencier toutes les semi-copules aspectuelles les unes par rapport aux autres (cf. 1. supra), le nœud du problème semble se trouver dans l'absence d'une classification détaillée, basée sur une étude de corpus, qui distingue entre d'une part les emplois avec et sans contrôle (et notamment la distinction entre sujet [ + animé ${ }^{28}$ et [- animé $\left.]\right)$ et, d'autre part, entre les emplois dynamiques et adynamiques (évidentiels). On trouve un reflet de cet amalgame dans la description de Camprubi (1982:67-68).

Notre analyse, qui contraste hacerse et se faire, pourrait à tort donner l'impression que hacerse se comporte un peu comme devenir, c'est-à-dire comme une espèce de verbe générique exprimant un changement d'état sous-spécifié. Toutefois, il faut signaler deux différences de taille: (i) $d e$ venir ne peut jamais exprimer la responsabilité-aussi insignifiante soitelle-du sujet; (ii) la portée de hacerse se voit cependant par la concurrence de verbes tels que ponerse et volverse (cf. 3.1.1.1.), dont l'analyse nous mènerait trop loin.

3.2.3. Les $\mathbf{S}(\mathbf{N})$. Terminons par quelques remarques concernant les noms attributs. Du côté des noms nus, il faut notamment relever les noms de type inanimé comme dans se faire vie, se faire substance, se faire loi, etc. (Frantext). Notamment les noms abstraits sont bien représentés (cf. Lauwers 2007): se faire réalité, se faire férocité. Si l'on compare avec l'espagnol, les noms nus sont 5 fois moins fréquents (1,76 vs 7,91 occ. par million de mots), ce qui ne fait que refléter une différence structurelle plus générale entre le français et l'espagnol, plutôt qu'une différence fondamentale entre se faire et hacerse. Ainsi on obtient du corpus Davies hacerse verdad, hacerse rutina, hacerse sonido, etc. Cet emploi relève, en général, d'un registre très littéraire et repose assez souvent sur des licences poétiques qui en rendent l'interprétation et, par conséquent, l'analyse linguistique, difficiles. Aussi n'attarderons-nous pas à ces emplois.

En revanche, ce qui nous semble plus pertinent pour l'analyse contrastive de hacerse et se faire, c'est les constructions impliquant des sujets (et attributs) animés. Contrairement à se faire, hacerse peut se combiner avec des sujets animés sans que ceux-ci contrôlent le procès (cf. se hizo borracho 3.1.1.2.2). Un tel emploi n'est pas possible avec se faire, qui impose toujours le contrôle. La même différence s'observe quant aux SN pleins, même s'il

28. Un constat analogue ressort déjà de Bybee et Eddington (2006), qui se limite aux sujets animés. En effet, on ne peut que constater que la plupart des adjectifs se rattachent à la configuration [+ animé] [- volonté] [+ intrinsèque]. 
est difficile de trouver des exemples attestés de SN animés impliqués dans un changement d'état dont le contrôle échappe au sujet de la phrase:

(48) Pedro se hizo el borracho más conocido del barrio

Les (S)N animés fournissent donc une preuve supplémentaire de la désagentivisation plus forte de hacerse (par rapport à se faire).

3.3. Les emplois statiques ou évidentiels: ' $\mathrm{X}$ se présente comme étant Y'. Jusqu'ici se faire et hacerse marquaient un changement d'état, voulu ou non, intrinsèque ou non intrinsèque. Il s'avère cependant que les deux verbes permettent encore un autre emploi, adynamique (ou statique), dont les caractéristiques sont, en plus de l'absence d'un pendant transitif, l'absence de changement d'état (d'où impossibilité d'y substituer le verbe devenir) et la présence d'un sujet inanimé. En voici un exemple:

(49) Incroyablement compact (de la taille d'une carte de crédit), il [= le téléphone portable] se fait très discret. ('est très discret, à mes yeux, il apparait comme discret')

(50) Deltia, el termostato, se hace discreto y elegante (www.teknoimport.cl/ Archivos_PDf/DELTA.../)

Ici, on ne peut plus détecter de changement d'état qui va de [-discret] à [+discret]. En revanche, on a affaire à un état de chose perçu par un expérienceur, le plus souvent le sujet parlant. Ainsi, le sens de hacerse/se faire peut être paraphrasé par 'paraître' ou 'apparaître comme'. Dans ce qui suit, nous allons aborder ce problème en détail, ainsi que les nuances qui séparent les deux verbes. Nous présenterons d'abord se faire (3.3.1), puis hacerse (3.3.2), pour terminer par quelques différences d'ordre morphosyntaxique (3.3.3).

3.3.1. Se faire évidentiel. Pour se faire, l'emploi évidentiel semble très récent. On n'en trouve aucun exemple dans Frantext qui soit complètement dépourvu de la dimension dynamique (il y a toujours un devenir, éventuellement un 'devenir pour X'). Pour trouver des exemples, il faut plutôt se tourner vers Google:

(51) [téléphone portable] En effet, le fin boitier en aluminium brossé du Shine KE970 sur lequel coulisse un immense écran $(\mathrm{Y})$ se fait très agréable lors de sa prise en main. (www.pixmania-pro.fr/fr/fr/lg/shineke970/494406/fiche.html)

On ne saurait cependant négliger l'importance numérique de cet emploi récent. La totalité des 30 exemples avec un sujet inanimé de se fait très discret sont de ce type, ainsi que deux des douze exemples de se fait très rare. En outre, 13 autres exemples ont été relevés dans un corpus qui, au total, n'en contient que 113 ("se fait très + ADJ"), animés et inanimés confondus. En tout, 45 des 113 exemples peuvent donc être rattachés au type adynamique évidentiel. Cependant, il est parfois difficile de savoir si l'on a encore affaire à un changement d'état ou non. D'où le concept de bridging context 
où les interprétations dynamiques et statiques semblent se superposer et où la réinterprétation peut avoir lieu. C'est ce qu'illustrent les exemples suivants (52):

(52) On rejoint alors la grosse N90 à l'entrée d'Andenne. Il ne nous reste plus qu'à rejoindre le centre de la ville. Nous terminons l'itinéraire sur le pont qui enjambe la Meuse. A cet endroit, elle se fait large et majestueuse.

Ainsi, dans l'exemple, la Meuse apparaît comme large et majestueuse, mais, comme la situation décrite s'insère dans un itinéraire parcouru par l'observateur-auquel correspond un déroulement temporel-, on peut encore y reconnaître un devenir: à partir de là, la Meuse devient large et majestueuse, alors qu'elle ne l'était pas plus en amont. On notera d'ailleurs que devenir peut encore se substituer à se faire, contrairement à l'exemple précédent.

Le verbe se faire s'inscrit dans le domaine de l'évidentialité dans la mesure où, avec se faire (et hacerse), le locuteur indique les sources de son savoir, les indices (= angl. evidence) sur lesquels son attitude, par rapport au contenu de la proposition, est basée (Dendale et Tasmowski 2001; Hassler 2002; Aikhenvald 2006; Ekberg et Paradis 2009). Pour notre propos, il suffira de rappeler ici que plusieurs types d'évidentialité ont été reconnus (Chafe 1986; Willet 1988) et que se faire (tout comme hacerse) se rattachent à l'évidentialité dite indirecte, plus particulièrement au type inférentiel (Willet 1988). Le locuteur infère une conclusion à partir d'indices sensoriels. Sous ce rapport, se faire se rapproche d'autres semi-copules telles que sembler, paraître/parecer, faire (elle fait vieille; Delplanque 2006; Lauwers 2008) et look (Miller 2009). Or, contrairement à ces verbes, l'inférence sur laquelle est basée la conclusion ne peut pas être annulée:

(53) (a) *Le boîtier se fait agréable lors de sa prise en main, mais il ne l'est pas (en réalité)

(b) vs. il \{fait / paraît\} vieux, mais il ne l'est pas (en réalité)

C'est qu'avec se faire la dimension évidentielle se cantonne au domaine de la manifestation sensorielle, sans que le sémantisme du verbe bascule dans les apparences, voire même dans le contrefactuel (Lauwers 2008:55). On peut donc parler d'un emploi évidentiel, qui fait penser un peu à l'évidentialité dite directe, où seule la perception (directe) de la réalité décrite est en jeu (Willet 1988) et où le calcul inférentiel est presque réduit à néant. Il s'ensuit que le locuteur ne pas nier ce qu'il vient de dire, sous peine de se contredire.

$\mathrm{Vu}$ le caractère évidentiel de l'emploi adynamique, les adjectifs qui se combinent avec se faire se rattachent de préférence au champ de la perception (auditive, visuelle, etc.). Ainsi, à part les nombreux exemples de rare et discret, Google nous en fournit encore 14 exemples. Dès que les adjectifs 
s'éloignent de la perception sensorielle, bon nombre de natifs les rejettent, comme l'énoncé suivant:

(54) Le lendemain matin, le réveil se fait très complexe. Après une soirée festive, les élèves ont bien des difficultés à se lever. (tele.ados.fr/news/staracademy-resume-week-end-21-22-octobre-2006_article3206.html)

Avant de terminer l'analyse du français, il convient de se poser la question de savoir si l'emploi évidentiel s'observe aussi avec des sujets humains (en combinaison avec des adjectifs qui admettent le contrôle). La réponse est négative. Certes, on trouve des exemples comme:

(55) Bashung se fait docile par rapport à la métrique de Manset dans son dernier album. ('il se montre-pour une fois-docile')

mais ceux-ci impliquent un changement temporaire voulu et se rattachent au type 'prendre un air, se montrer' (cf. 3.1.2.1). ${ }^{29}$

3.3.2. Hacerse évidentiel. Par rapport à se faire (dont on ne trouve aucun exemple dans Frantext), l'emploi adynamique de hacerse est beaucoup plus fréquent. Ainsi, dans le corpus de Davies, on trouve 79 exemples d'emplois adynamiques strictement parlant, c'est-à-dire des exemples qui ne présentent aucun déroulement temporel menant d'un état $\mathrm{A}$ à un état B (16,2 pour cent des attributs adjectivaux, soit une fréquence de 5,12 occ. par million de mots). Cette interprétation assez stricte du trait [-dynamique] nous a permis d'identifier les cas adynamiques indiscutables. De même qu'en français, les bridging contexts sont légion:

(56) desdoblamiento de una mujer no encuentra sitio en la cabeza humana mientras no se hace evidente la rotundidad delatora

Cet exemple peut être paraphrasé par 'le dédoublement d'une femme n'entre pas dans la tête des humains tant que la rondeur ne 'devient ou n'est (paraît) évidente' (à propos d'une femme enceinte). L'emploi évidentiel n'est pas seulement plus fréquent, il admet aussi une gamme d'adjectifs beaucoup plus large:

(57) y no se me hace bueno que cortaras mis créditos, pero tu sabrás (www .youtube.com/watch?v=UCWbbevpyTc\&feature=related)

De manière globale, le sens de hacerse correspond à celui de se faire, ce qui explique aussi pourquoi tous les exemples adynamiques de se faire peuvent être traduits littéralement en espagnol. Hacerse, tout comme se faire, se rapproche fortement de 'être' auquel il ajoute une nuance évidentielle ('et

29. Le type Pierre se fait vieux ne peut pas non plus être considéré comme un emploi évidentiel pour la simple raison qu'il renvoie toujours à un changement d'état, même si certains locuteurs tendent à y voir par surcroît un aspect évidentiel ("ça se voit"), peutêtre sous l'influence de il fait vieux. 
cela se voit'), comme nous l'avons vu sous 3.3.1. De même, l'interprétation contrefactuelle est carrément exclue:

(58) *El valle del Motagua se hace singular [. . .], pero en realidad no lo es

Il n'empêche que le spectre d'emploi de hacerse dans le domaine adynamique se voit élargi par l'insertion d'un datif, qui semble orienter l'interprétation vers 'parecer', comme le montrent aussi certains indices contextuels ("no muy larga”, "pareció”, "pronto”):

(59) Las calles se le hicieron misteriosas y el edificio del Multifamiliar le pareció de pronto oscuro

Dans certains exemples, cette interprétation déteint sur l'emploi sans datif:

(60) (a) El trayecto por los subterráneos no se hizo largo porque la visión de los tesoros desató la lengua de Balbina (Davies, $20^{\mathrm{e}} \mathrm{s}$.)

(b) 'Le trajet ne parut pas long, parce que ...'

On peut dès lors visualiser le positionnement de hacerse et se faire sur une échelle qui va du réel au contrefactuel:

Figure 3

Réel

Contrefactuel

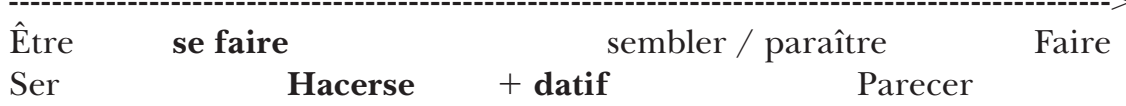

En d'autres termes, hacerse semble plus éloigné du pôle 'être' et plus proche du pôle 'paraître' que se faire, mais ne se confond pas pour autant avec parecer. Par ailleurs, l'espagnol ne dispose pas d'un verbe comme faire (il fait vieux), qui, sémantiquement, correspond presque toujours à un contrefactuel.

Une autre différence entre se faire et hacerse concerne les types de sujets. En général, les deux verbes se construisent avec un sujet inanimé, mais hacerse, du moins dans l'espagnol du Mexique, semble admettre des sujets animés en emploi évidentiel:

(61) $\mathrm{Y}$ a ti se te hacen buenos los mexicanos? (Habla Culta: Mexico: M31; Davies)

3.3.3. (Morpho-)syntaxe. L'analyse contrastive des emplois évidentiels de hacerse et se faire fait aussi apparaître des différences morphosyntaxiques.

Tout d'abord, hacerse admet un datif qui réfère à un expérienceur précis. A défaut, l'expérienceur reste indéterminé comme en français, mais 
peut très souvent être récupéré contextuellement ou situationnellement (notamment le sujet parlant):

(62) Tras una espera no muy larga pero que a ella se le hacía interminable, aparecía él (Davies)

En français, la construction dative n'est pas possible pour des raisons structurelles. Se faire obéit en effet à un mécanisme de blocage plus général concernant les "groupements interdits" de pronoms, qui figure dans toutes les grammaires de référence. ${ }^{30} \mathrm{D}$ 'autre part, l'échappatoire par le biais des pronoms toniques n'est pas disponible pour se faire, quelle que soit son interprétation:

(63) *cela se fait bizarre à lui / à Paul

Rappelons que la construction à datif expérienceur est typique des verbes évidentiels comme sembler, paraître, etc. On pourrait tenter l'hypothèse selon laquelle la disponibilité de la combinaison datif $+\mathrm{SE}$ a favorisé le développement d'un emploi évidentiel en espagnol. Cette possibilité de construction y existe en effet depuis le $15^{\mathrm{e}}$ siècle. Ainsi, dans le corpus de Davies, on trouve 78 exemples de la construction dative avant 1600, dont 21 (27 pour cent) sont adynamiques, c'est-à-dire une fréquence absolue de 2,15 occ. par millions de mots:

(64) una vez se me hazia estraño su parecer otra vez me parecia como que le conocia sin poder le conocer (Davies)

Les indices contextuels montrent clairement que seule la dimension évidentielle est en jeu. On ne saurait cependant croire que la construction dative exclue complètement la lecture dynamique ni avant 1600, ni de nos jours. En gros, un tiers des exemples dans le corpus de Davies $\left(20^{\mathrm{e}} \mathrm{s}\right.$.) $(10 /$ 32) et un peu plus d'un tiers pour le corpus datif d'avant 1600 sont de type statique (au sens strict du terme, cf. notre définition très stricte point 3 et 3.3); le reste est toujours dynamique.

La deuxième différence de taille entre hacerse et se faire est que les emplois évidentiels de hacerse entrent dans la construction impersonnelle à complétive et à infinitif extraposés, constructions également typiques des verbes évidentiels (Miller 2009, se référant à Gisborne 2000):

(65) Se nos hacía más llevadero pasar toda una jornada encerrados www .centrogarrigues.com/ficherosceg/asociapdf/asocia_16.pdf

Se faire, par contre, ne permet pas cette construction:

(66) *Il se fait \{évident / nécessaire / etc.\} que Y / de Y

30. "Les pronoms conjoints $m e$, te, se, nous et vous ne peuvent pas (sauf les pronoms explétifs: §647) se trouver juxtaposés deux à deux, ni se joindre aux pronoms lui et leur" (Grevisse et Goosse 1993:1044). 
Notons que la construction avec complétive extraposée semble être un régionalisme, cantonné essentiellement à l'espagnol du Mexique, comme nous le signale un relecteur anonyme. Comme elle passe pour un indice d'une transparence plus grande (cf. aussi Tobback et Lauwers à par. concernant se révéler vs s'avérer), la variation géographique interne au domaine hispanique illustre très bien l'idée que la grammaticalisation est un processus graduel qui ne se déroule pas toujours au même rythme, comme le montre d'ailleurs aussi la comparaison du français et de l'espagnol.

Sur le plan morpho-sémantique, on peut s'attendre à de fortes restrictions aspectuo-temporelles dues à la décatégorialisation qu'entraîne l'apparition d'emplois évidentiels (Hopper et Traugott 1993:103; Seuren 2003; Cornillie 2007, ch.3). On notera ainsi que dans cet emploi se faire perd ses temps accomplis (a), alors que dans ses emplois dynamiques, se faire, (b) autorise encore le passé composé résultatif marquant le résultat du changement (abouti):

(67) (a) *La MX-5 s'est faite très bruyante. (Google)

(b) l'Arema s'est faite très discrète depuis la crise politiqueY (Google)

En espagnol, les restrictions portant sur l'accompli semblent inexistantes, comme le montre déjà l'exemple cité par Porroche (1990:116). Quant au futur simple, par contre, il ne semble pas offrir de restrictions:

(68) Logé dans un boîtier très compact, il se fera très discret dans une poche ou dans une sacoche

Même le futur périphrastique reste possible. Nous avons trouvé quelques exemples dans Google, tant pour l'emploi dynamique que statique:

(69) Mathieu a récupéré son sac, on retourne à la voiture, puis on met le cap sur le camping. Le retour va se faire très calme car Mary se tape sa migraine.

Mais ils restent tout de même très limités: sur les 531 exemples de "va se faire très", attestés sur Google, nous avons recueilli 24 exemples de se faire + adjectif.

4. Bilan et discussion: autonomisation (lexicalisation) allant de pair avec un processus de grammaticalisation et de subjectification, aboutissant à la superposition d'emplois. L'analyse étant faite, il reste à mettre les résultats en perspective. Dans un premier temps, nous prendrons un peu de hauteur pour établir les profils des deux verbes (4.1). Ensuite, nous situerons ces deux profils à la lumière de deux problématiques: l'autonomisation (l'intransitivisation / lexicalisation) de la construction pronominale, qui aboutit à la création d'une nouvelle copule (4.2), et la théorie de la subjectification (4.3). Ces deux facteurs ont abouti à la superposition d'emplois ou de sens (polysémie, voire hétérosémie) dans une seule forme de surface (4.4). 
4.1. Deux faux amis. Tout d'abord, que conclure de cette analyse en miroir de deux verbes qui se sont révélés de redoutables faux amis? Voici les principales divergences:

$1^{\circ}$. De manière globale, se faire + adjectif est en recul (cf. fig.1 +2 ), ce qui explique pourquoi dans certains secteurs du spectre sa présence est désormais limitée à quelques survivances lexicalisées: [SN animé] se faire beau/joli et [SN animé] se faire vieux. Cela explique aussi pourquoi, de nos jours, bon nombre des exemples littéraires provenant de Frantext sont rejetés par les locuteurs natifs. De son côté, Hacerse + Adj. a désormais un emploi très large, qui n'a cessé de gagner du terrain.

$2^{\circ}$. Les deux verbes connaissent, en plus des emplois dynamiques, un emploi adynamique de type évidentiel, analogue, mais pas tout à fait identique (cf. 4.3).

$3^{\circ}$. De manière globale, se faire est davantage lié à l'exercice de contrôle par le sujet (plus 'agentif') que hacerse, même si les deux verbes ont connu un recul très spectaculaire pour ce qui est du taux de sujets animés depuis le $16^{\text {e }}$ siècle (2). Il s'ensuit que dans le domaine de l'animé la distribution des deux verbes est assez inégale. Ainsi, nous avons observé l'absence du type elle se fait belle (3.1.1) et de l'interprétation 'prendre un air, se montrer' pour hacerse; l'espagnol préfère en effet ponerse (3.1.2.1). D'autre part, nous avons relevé l'absence du changement intrinsèque (voulu) avec se faire, contrairement aux stades plus anciens de la langue et au domaine nominal (se faire moine). En plus, hacerse peut facilement être utilisé avec des sujets animés sans que s'impose une interprétation 'contrôlée', tant avec des adjectifs (Mi abuela se hace pequeña con la vejez; 3.2.2; Pedro se hace desagradable), qu'avec des noms (se hace borracho; 3.1.1.2). Pour se faire, par contre, cela n'est possible qu'avec se faire vieux. Ceci montre que le peu d'exemples de hacerse avec sujet animé ne sont, qui plus est, pas nécessairement associés à l'idée de contrôle. Sur ce point, l'examen d'un corpus monolingue, par contraste avec l'étude de se faire, a mis le doigt sur un aspect mal théorisé de la grammaire espagnole (cf. 1.; 3.2.2): si hacerse peut être associé à l'exercice de la volonté, c'est uniquement par opposition à volverse et dans le domaine animé, car, en soi, hacerse autorise les deux emplois, volitifs (Pedro se hace rico) et non volitifs (Pedro se hace desagradable [sin querer]). Une typologie plus nuancée des emplois s'impose, car l'opposition [+/- contrôle], qui correspond en partie à l'opposition [+/- animé], s'est avérée très pertinente pour la traduction de hacerse en français:

(70) (a) Las personas / las cosas que se hacen visibles

(b) *Les personnes qui se font visibles ('deviennent')

(c) Les choses qui se font visibles

En effet, avec les animés, se faire marque un changement contrôlable, alors que les exemples avec hacerse permettent à la fois des changements contrôlables et non contrôlables. Dans le domaine des inanimés, enfin, 
d'autres règles plus subtiles sont d'application, qui ont partie liée avec la subjectification.

$4^{\circ}$. Dans le domaine adjectival, se faire ne se prête pas à l'expression du changement intrinsèque; on a toujours un changement superficiel voulu, soit physique, soit comportemental (d'où l'interprétation 'adopter un air'). Ce contraste est moins clair dans le domaine nominal (se faire moine), encore que se faire le $\mathrm{SN}$ semble lié à l'idée d'une appropriation illégitime (et donc moins intrinsèqueque dans le cas de devenir / hacerse) d'un statut (cf. 3.1.2.3).

$5^{\circ}$. La mise en rapport avec la construction transitive correspondante est devenue problématique dans bon nombre de cas. Cela vaut d'autant plus pour se faire que la construction attributive transitive (à attribut du COD) est désormais confinée à quelques emplois marginaux. Ici faire a cédé devant rendre. Nous approfondirons cet aspect sous 4.2.

4.2. Autonomisation. A la suite de la disparition de faire + ACOD, le lien entre la construction transitive et la construction pronominale (réfléchie ou passive) a été coupé. Celle-ci ne peut donc plus être considérée comme une reformulation productive (selon un procédé syntaxique productif) du tour transitif. Du coup, la construction pronominale s'est autonomisée. Elle concerne désormais un verbe (ou une construction) essentiellement pronominal(e), c'est-à-dire lexicalisé dans son emploi copule. Ce lien persiste cependant, dans les emplois décrits sous 3.1.1 (elle se fait belle; il se fait président), encore que la mise en rapport de se faire moine avec on l'a fait moine devienne déjà plus délicate.

Cela ne devrait pas nous étonner, car, dans la littérature sur la construction pronominale, on a noté une certaine tendance à l'autonomisation qui s'inscrit dans la nature même de la construction pronominale (réfléchie). On ne saurait sous-estimer les effets de sens provoqués par la construction pronominale. Ainsi, celle-ci tend à rapprocher le rôle de l'observateur (l'agent) et de l'observé (le patient) (Les habitants de ces territoires se reconnaissent en vous) alors que le tour transitif distingue clairement les deux rôles (J'ai reconnu mon père). On notera aussi que, dans certains cas, seul le tour pronominal admet la construction attributive: il se reconnaît coupable / *on l'a reconnu coupable (Melis 1990:64-65), ce qui est un autre indice d'une tendance à l'autonomisation du tour pronominal.

Appliquons ce constat au verbe hacerse, qui, lui, a encore un pendant transitif à attribut du COD:

(71) (a) este acontecimiento lo hizo rico

(b) su tío lo hizo rico

(c) Juan se hizo rico

Dans la construction transitive, le changement d'état est provoqué par une personne qui a fait don d'une somme importante ou par un événement extérieur qui a procuré une certaine richesse à Jean. On a affaire à deux ac- 
tants bien différenciés, un agent et un bénéficiaire. En revanche, le sens de la construction réfléchie, qui, certes est encore analysable syntaxiquement, ne peut pas être paraphrasé par 'Jean a donné une somme importante à lui-même qui l'a rendu riche'. Il y a comme une superposition de rôles (un processus de clôture actancielle) qui à la fois affaiblit le caractère agentif du sujet (une simple responsabilité, volonté) et affecte son rôle comme patient, qui, en effet, est moins profilé, moins autonome par rapport au rôle d'agent. Le développement d'un sémantisme autonome (et d'une certaine autonomie syntaxique débouchant sur la création d'un verbe intransitif) est donc tout à fait courant dans le domaine des tours pronominaux.

Regardons maintenant la typologie des effets de sens à la lumière de ce processus d'autonomisation. En l'absence d'une étude diachronique poussée, nous devons nous limiter ici à évoquer quelques liens ou connexions entre les divers sens générés par la construction $s e+$ faire qui sont autant d'étapes "logiques" entre les différents sens de la combinaison.

(i) se faire + Adj / N: 'exercer une pression/action sur soi-même pour devenir X' (se faire beau / petit; se faire président/se faire les héros de la résistance)

$>$ (ia) 'devenir par un acte voulu' (se faire moine)

$>$ (ib) 'afficher un état d'âme temporaire X, prendre un air X [non intrinsèque]' (se faire méchant), puis animé > inanimé (par métonymie): sa voix se fit grave

(ii) extension vers les inanimés

En clair, au cours de ce processus d'autonomisation, l'agentivité de la construction (liée au sens factitif inhérent au sémantisme de faire/hacer) s'est affaiblie, tout comme le profilage de deux rôles différencies sous la pression de l'effet de clôture propre aux constructions pronominales. Concrètement, on peut supposer que, dans un premier temps, le référent dénoté par le sujet ait gardé encore une certaine responsabilité dans le procès (cf. se faire écraser par une voiture vs être écrasé par une voiture), avant que la construction ne s'étende à des sujets inanimés, pour se vider complètement de son agentivité. Ce dernier processus a été poussé beaucoup plus loin en espagnol. Comme les évolutions en question remontent très loin dans le temps, il n'a pas été possible d'attester ces évolutions diachroniquement. Par ailleurs, une deuxième piste pourrait être envisagée (à l'instar de quelques autres semi-copules), à savoir l'autonomisation de se faire / hacerse (intransitif) par rapport à faire / hacer. ${ }^{31}$

Le résultat de ce processus d'autonomisation ou-si on se place au niveau de chacun des lexèmes du lexique-de lexicalisation est la créa-

31. Ici s'impose une analyse approfondie des premiers emplois intransitifs de se faire. Nous avons ici affaire à deux "voies" dans la création de verbes copules (autonomisation par rapport à une construction à attribut de l'objet vs intransitivisation suivie d'incorporation valencielle d'un attribut périphérique). Le lecteur se reportera à Lauwers et Tobback (soumis pour publication). 
tion d'une nouvelle (semi-)copule, certes multistratale. Comme le résultat est un "outil grammatical" (par analogie avec l'auxiliarisation) et que le processus transforme le pronom réfléchi en une sorte de morphème grammatical marquant la clôture actancielle, on pourrait aussi parler de grammaticalisation. Ainsi, se faire et hacerse rejoignent le paradigme des semi-copules à base pronominale qui comprend s'avérer, se révéler, se montrer, s'annoncer, etc. (Tobback et Lauwers, à par.). Ici, lexicalisation et grammaticalisation vont de pair (cf. Fagard et de Mulder 2007 à propos du développement de préposition complexes).

4.3. Evidentialité et subjectification. Enfin, notre analyse a fait ressortir que, tout récemment, se faire a développé un emploi évidentiel adynamique nouveau, qui se distingue cependant de l'emploi évidentiel de hacerse, qui existe depuis 5 siècles. On a pu constater qu'au niveau du sens, se faire exprime une valeur évidentielle qui se rapproche d'une certaine façon de l'évidentialité dite directe, alors que hacerse tend plus vers le paraître, sans pour autant se confondre avec celui-ci comme le montre la. En outre, l'emploi de se faire est cantonné à un secteur lexical particulier, alors que de telles restrictions lexicales ne s'observent pas pour hacerse, dont l'emploi évidentiel s'étend par ailleurs jusqu'aux sujets animés. Du point de vue de la construction, hacerse a emprunté, à la catégorie des verbes évidentiels, à la fois la construction dative et la construction impersonnelle à extraposition, ce qui est signe d'une meilleure intégration (3.3).

Si l'on met les résultats de cette étude en perspective, c'est comme si le recul de l'emploi dynamique de se faire (cf. 4.2), qui est allé de pair avec un cantonnement dans un certain nombre de secteurs lexicaux "subjectifs" (et une désagentivisation globale), avait préparé le terrain à un emploi adynamique et évidentiel. En effet, les emplois dynamiques (en combinaison avec un adjectif) se caractérisent souvent par une perspectivation subjective ('devenir pour quelqu'un') comme le montre l'analyse des adjectifs. Le changement d'état (emploi dynamique) se voit donc ramené à la sphère du sujet observateur (expérienceur). C'est pourquoi nous avons parlé ici de perspectivation subjective. Par ailleurs, les bridging contexts ne manquent pas, de même qu'en espagnol. On peut supposer qu'en espagnol la construction dative a accéléré le développement d'un sens évidentiel, qui a déteint ensuite sur la construction sans datif. Pour vérifier cette hypothèse, il faudrait une étude diachronique poussée du verbe hacerse, impliquant une analyse contextuelle approfondie.

4.4. La superposition d'emplois. Le résultat de toutes ces évolutions est une polysémie, voire même hétérosémie (Lichtenberk 1991); dans laquelle on peut distinguer différentes strates. Premièrement, il y a un hacerse / se faire qui résulte de l'application d'une règle productive de pronominalisation qui aboutit à une interprétation réfléchie. Deuxièmement, cette même forme "de surface" abrite, en rapport à la fois avec le sémantisme du sujet (ainsi que de l'attribut) et la morpho-syntaxe, tantôt un chan- 
gement contrôlé intransitivé (3.1.2), tantôt un changement non contrôlé (3.2), tantôt un emploi adynamique évidentiel (3.3). Face à cette superposition de strates, ne faudrait-il pas reconnaître deux ou trois verbes se faire / hacerse? Le lien (diachronique) entre les emplois dynamiques et adynamiques étant clair (processus de subjectification), comme en témoignent les nombreux bridging contexts, il est légitime de traiter les emplois intransitifs (dynamiques et adynamiques) de hacerse/se faire sous l'angle de la polysémie. Par contre, les emplois qui connaissent encore un pendant transitif (3.1.1) devraient en toute logique être rattachés aux emplois (attributifs) du verbe non pronominal, respectivement faire et hacer. L'effet de clôture qu'on y observe parfois (hacerse rico; se faire moine) pourrait être mis sur le compte de la construction pronominale, qui se présente comme une variante diathétique de la construction transitive.

Peter Lauwers

Université de Gand et Université de Leuven

Claude Duée

Université de Castilla-La Mancha

\section{Dictionnaires et corpus}

Moliner, Maria. 1984. Diccionario de uso español. Madrid: Gredos.

Trésor de la Langue française. http://atilf.atilf.fr/tlf.htm.

Dictionnaire de l'Académie (édition 1692) http://gallica.bnf.fr/.

Davies, Mark. 2002-. Corpus del Español (100 million words, 1200s-1900s). http:// www.corpusdelespanol.org.

Frantext. http://www.frantext.fr/.

\section{Références}

AIKHENVAld, AleXandra. 2006. Evidentiality. Oxford: Oxford University Press. ATHANASIADOU, ANGELIKI, COSTAS CANAKIS et BERT CORNILlie, eds. 2006. Subjectification: Various Paths to Subjectivity. Berlin \& New York: Mouton de Gruyter.

BoUZet, JEAn. 1990. Grammaire espagnole. Paris: Belin.

Bybee, JOAN et DAVID EDdington. 2006. "A Usage-based Approach to Spanish Verbs of 'becoming'”. Language 82:323-355.

CAMPRUBI, MICHEL. 1982. Etudes fonctionnelles de grammaire espagnole. Toulouse: France Ibérie Recherche.

CHAFE, WAllace L. 1986. "Evidentiality in English Conversation and Academic Writing”. In Evidentiality: The Linguistic Coding of Epistemology, edd. Wallace L. Chafe et Johanna Nichols, 261-272. Norword, New Jersey: Ablex Publishing Corporation.

CORNILlie, BERT. 2007. Evidentiality and Epistemic Modality in Spanish (Semi-)Auxiliaries: A Functional-Pragmatic and Cognitive-Linguistic Approach. Applications of cognitive linguistics, 5. Berlin \& New York: Mouton de Gruyter. http://site .ebrary.com/lib/princeton/Doc?id $=10256480$ 
COSTE, JEAn et AUgustin Redondo. 1965. Syntaxe de l'espagnol moderne. Paris: Sedes.

DELPLANQUe, ALAin. 2006. "Juger d'après les apparences: le cas du français". Corela. Numéro spécial: Les verbes d'apparence. http://corela.edel.univ-poitiers.fr/ document.php?id $=838$

DENDALE, PATRICK et LILIANE TASMOWSKi. 2001. "Introduction: Evidentiality and Related Notions". Journal of Pragmatics 33:339-348.

EDdington, DAvid. 1999. On 'becoming' in Spanish: A Corpus Analysis of Verbs Expressing Change of State. http://linguistics.byu.edu/faculty/eddingtond/become. html

EKBERG, LENA, et CARITA PARADIS. 2009. "Evidentiality in Language and Cognition”. Functions of Language 16:5-7.

FAGARD, BENJAMIN et WALTER DE MULDER. 2007. "La formation des prépositions complexes: grammaticalisation ou lexicalisation?". Langue française 156:9-29.

GISBORNE, NiCOLAS. 2000. "The Complementation of Verbs of Appearance by Adverbs". In Generative Theory and Corpus Studies: A Dialogue from 10 ICEHL, edd. Ricardo Bermúdez-Otero et alii, 53-75. Topics in English Linguistics, 31. Berlin \& New York: Mouton.

GREVISSE, MAURICE et ANDRÉ GOOSSE. 1993. Le bon usage: grammaire française. $13^{\mathrm{e}}$ éd. rev. Paris: Duculot.

HASSLER, GERDA. 2002. "Evidentiality and Reported Speech in Romance Languages”. In Reported Discourse. A Meeting Ground for Different Linguistic Domains, eds. Tom Güldemann et Manfred von Roncador, 143-172. Amsterdam \& Philadelphia: Benjamins.

Hengeveld, KeEs. 1992. Non-Verbal Predication: Theory, Typology, Diachrony. Berlin \& New York: Mouton de Gruyter.

hopper, PAUl et Elisabeth Closs traugott. 1993. Grammaticalization. Cambridge: Cambridge University Press.

LAMIROY, BÉATRICE et LUDO MELIS. 2005. "Les copules ressemblent-elles aux auxiliaires?”. In Les périphrases verbales, edd. Hava Bat-Zeev Shyldkrot et N. Le Querler, 145-170. Amsterdam: Benjamins.

LANGACKER, RONALD w. 1990. "Subjectification". Cognitive Linguistics 1:5-38.

LAUWERS, PETER. 2007. "Les noms nus inanimés attributs. Essai de classification syntaxique et sémantique”. Journal of French Language Studies 17:151-171. . 2008. "Les emplois attributifs de faire". Studia Neophilologica 80:43-64.

LAUWERS, PETER, GUDRUN VANDERBAUWHEDE et STIJN VERLEYEN. (à par.). "How False Friends Give True Hints about Pragmatic Markers". Languages in Contrast.

LAUWERS, PETER et ElS TOBbACK. (soumis pour publication). "Les verbes attributifs: inventaire(s) et statut(s)". Langages.

LeEman, DANiElle. 1992. "Deux classes d'adjectifs en -ble ?". Langue française 96:44-64.

LiCHTENberk, FRAntisek. 1991. "Semantic Change and Heterosemy in Grammaticalization”. Language 67:475-509.

MELIS, LUDO. 1990. La voie pronominale. La systématique des tours pronominaux en français moderne. Louvain-la-Neuve: Duculot.

Miller, PHilip. 2009. "Prédication et évidentialité: de l'emploi copule des verbes de perception en anglais". Faits de langue 31/32:253-262.

PORRoche ballesteros, marguerita. 1990. Aspectos de la atribución en español. Zaragoza: Libros Pórtico.

SEuren, Pieter. 2003. "Verb Clusters and Branching Directionality in German and Dutch". In Verb Constructions in German and Dutch, edd. Pieter A. M. Seu- 
ren et G. Kempen, 247-296. Amsterdam studies in the theory and history of linguistic science. Series IV. Current issues in linguistic theory, 242. Amsterdam \& Philadelphia: Benjamins.

STEIN, DIETER et SUSAN WRIGHT, éds. 1995. Subjectivity and Subjectivisation: Linguistic Perspectives. Cambridge: Cambridge University Press.

TOBBACK, Els et PETER LAUWERs. (à par.). "Une analyse en miroir de deux verbes évidentiels: s'avérer vs se révéler". Revue Romane.

traugott, elisabeth Closs. 1989. "On the Rise of Epistemic Meanings in English: An Example of Subjectification”. Semantic Change. Language 65:31-55.

VAN PETEGHEM, MARLEEN. 1993. La détermination de l'attribut nominal. Étude comparative de quatre langues romanes (français—espagnol_italien—roumain). Bruxelles: Palais des Académies, AWLSK, Classe des Lettres.

willems, DominiQue. 1981. Syntaxe, lexique et sémantique: les constructions verbales. Gent: Rijksuniversiteit Gent.

willet, THOMAS. 1988. "A Cross-Linguistic Survey of the Grammaticization of Evidentiality”. Studies in Language12:51-97. 